\title{
Deformable Model-based Image Registration *
}

\author{
Jundong liu \\ School of Electrical Engineering and Computer Science \\ Ohio University
}

Athens $\mathrm{OH} 45701$

\section{Introduction}

Medical image analysis technology, with image segmentation, image matching /registration, motion tracking and the measurement of anatomical and physiological parameters as the main research areas, has seen a tremendous amount of growth over the past decade. The work described in this chapter is concerned with the problem of automatically aligning $3 \mathrm{D}$ medical images.

Image registration is one of the most widely encountered problems in a variety of fields including but not limited to medical image analysis, remote sensing, satellite imaging, optical imaging, etc. A possible definition of this problem is: determine the coordinate transformation, or mapping, relating different views of the same or similar objects. These views may arise from:

- The same object "imaged" with different sensors.

- The same object "imaged" repeatedly with the same sensor.

- Multiple similar objects all "imaged" with the same sensor.

- A single object "imaged" with a sensor, and a model (matching)

Medical imaging plays a very important role in modern clinical practice. Often, a single modality alone can not provide adequate information about a patient's condition, so they are imaged by a second and sometimes more modalities. Different modalities, as shown in Figure 1 (from the homepage

*Draft, Jan. 22, 2006 

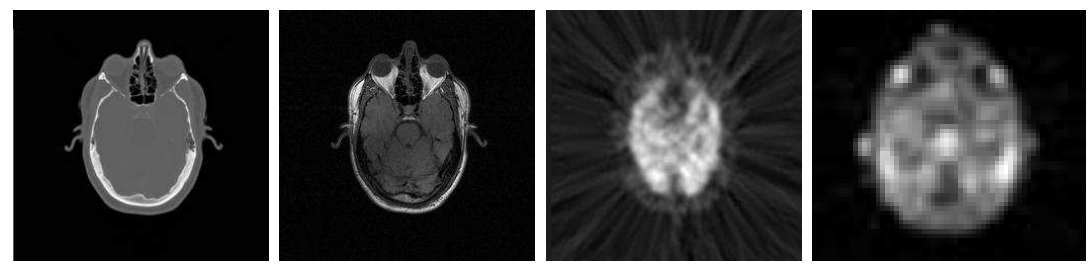

Figure 1: Image from different modalities, from left to right: CT, MR, PET, SPECT (Available online at http://www.isi.uu.nl/Research/Registration/)

of the Image Science Institute at the Univeristy of Medical Center Utrecht. Available online at http://www.isi.uu.nl/Research/Registration/registrationframe.html), can usually provide different, complementary or partially overlapping aspects of the anatomy under examination. So if the information from them can be combined, it will greatly facilitate the clinicians to perform surgical planning, diagnosis and treatment. Among the widely used modalities, X-ray, CT (computed tomography), MRI (magnetic resonance imaging) and US (ultrasound) depict anatomical information, while PET (positron emission tomography), SPECT (single-photon emission computed tomography), fMRI (functional MRI) provide information on the metabolism of the underlying anatomy.

Figure 1 shows images from 4 different modalities: CT, MR, PET, SPECT, illustrating the different types of information they contain. As we can see from the figure, CT is sensitive to bone density, MR to tissue density whereas PET and SPECT depict the physiology.

Figure 2 (from the homepage of the Image Science Institute Department of medical Imaging. Available online at http://www.isi.uu.nl/Research/Registration/ registration-frame.html) also shows how multi-modal data can be used to provide a better understanding of physiology of the human brain aided by the presence of precise anatomical information. It shows the right and left hemispheres of a brain, segmented from an MR image. Information from a SPECT scan is overlayed on the cortex. Color encoding is used to indicate the amount of cerebral blood perfusion: from light gray for low perfusion, via yellow, to red for high perfusion. As we can see, the picture shows an area with increase blood perfusion in the right hemisphere and there is indicative of a pathology on the right hemisphere. 


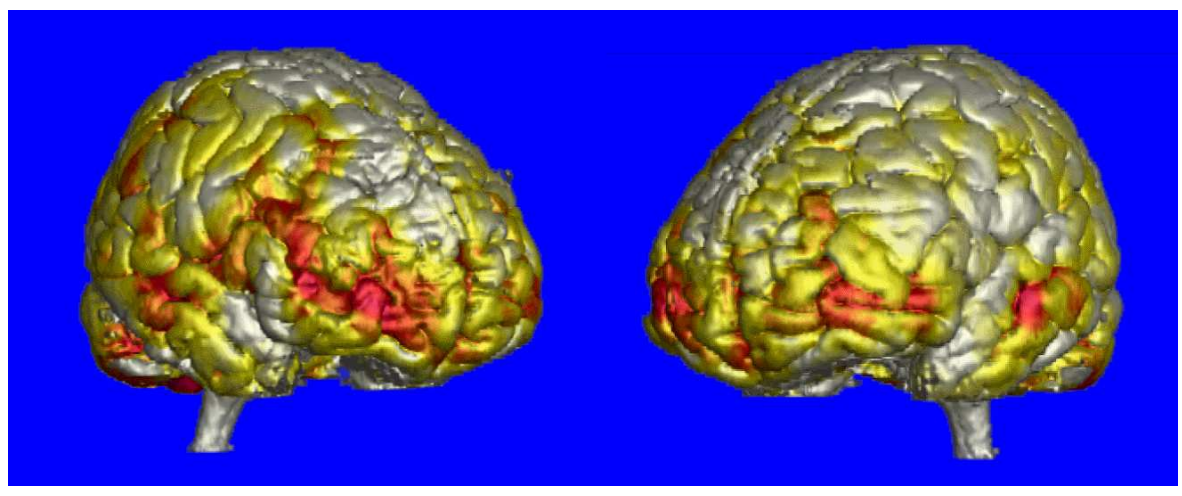

Figure 2: An example of a multimodal visualization (Available online at http://www.isi.uu.nl/Research/Registration/)

However, multi modality images are usually acquired with different devices, at different times, so inevitably there will be some motion between them. This makes accurate geometrical registration a prerequisite step for the effective fusion of the information from multi modality images.

\subsection{Mathematical Definition}

Let $I_{1}(x, y, z)$ and $I_{2}(x, y, z)$ denoted two images. The relationship between these two images can be expressed as

$$
I_{2}(x, y, z)=g\left(I_{1}(T(x, y, z))\right)
$$

where $\mathrm{T}$ is a $3 \mathrm{D}$ spatial-coordinate transformation and $\mathrm{g}$ is an intensity transformation.

The registration problem is to find the optimal spatial and intensity transformation so that the images are matched well. Finding the parameters of the optimal geometric coordinate transformation is generally the key to any registration problem while the intensity transformation is not always the task interest.

The transformations can be classified into global and local transformations. A global transformation is given by a single equation which maps the entire image. Local transformations map the image differently depending on the spatial location and are thus more difficult to express succinctly. The most common global transformations are rigid, affine and projective transformations. 
A transformation is called rigid if the distance between points in the image being transformed is preserved. A rigid transformation can be expressed as:

$$
\left\{\begin{array}{l}
u(x, y)=\left(\cos (\phi) x-\sin (\phi) y+d_{x}\right)-x \\
v(x, y)=\left(\sin (\phi) x+\cos (\phi) y+d_{y}\right)-y
\end{array}\right.
$$

where $u(x, y)$ and $v(x, y)$ denote the displacement at point $(x, y)$ along the $X$ and $Y$ directions; $\phi$ is the rotation angle, and $\left(d_{x}, d_{y}\right)$ the translation vector.

A transformation is called affine when any straight line in the first image is mapped onto a straight line in the second image with parallelism being preserved. In $2 \mathrm{D}$, the affine transformation can be expressed as:

$$
\left\{\begin{array}{l}
u(x, y)=\left(a_{11} x+a_{12} y+d_{x}\right)-x \\
v(x, y)=\left(a_{21} x+a_{22} y+d_{y}\right)-y
\end{array}\right.
$$

where

$$
\left[\begin{array}{ll}
a_{11} & a_{12} \\
a_{21} & a_{22}
\end{array}\right]
$$

denotes an arbitrary real-valued matrix. Scaling transformation, which has a transformation matrix of $\left(\begin{array}{cc}s_{1} & 0 \\ 0 & \hat{s}_{2}\end{array}\right)$ and shearing transformation, which has a matrix of $\left(\begin{array}{cc}1 & s_{3} \\ 0 & 1\end{array}\right)$ are two examples of affine transformation, where $s_{1}, s_{2}$ and $s_{3}$ are positive real numbers.

A more interesting case, in general, is that of a planar surface in motion viewed through a pinhole camera. This motion can be described as a $2 \mathrm{D}$ projective transformation of the plane

$$
\left\{\begin{array}{l}
u(x, y)=\frac{m_{0} * x+m_{1} * y+m_{2}}{m_{6} * x+m_{7} * y+1}-x \\
v(x, y)=\frac{m_{3} * x+m_{4} * y+m_{5}}{m_{6} * x+m_{7} * y+1}-y
\end{array}\right.
$$

where $m_{0} \ldots m_{7}$ are the global parameters.

In clinical practice, the most commonly used global registration transformations are rigid and affine. For the brain images taken from the same patient using different devices, e.g, CT/MR, or the same devices but at different times, usually a rigid transformation is adequate to explain the variation between them. When two images depicting the same scene are taken from the same viewing angle but from different positions, i.e, the camera was zoomed in/out, or rotated around its optical, an affine transformation is required to match these two images. In this thesis, we will be mainly dealing with these type of global transformations. 
When a global transformation does not adequately explain the relationship of a pair of input images, a local transformation may be necessary. To register an image pair taken at different times with some portion of the body experienced growth, or to register two images from different patients falls into this local transformation registration category. A motion (vector) field is usually used to describe the change/displacement in local transformation problem.

\subsection{The Challenges Remaining}

In the past two decades, a broad range of techniques has been developed to deal with the image registration problem for various types of data. For a comprehensive review, we refer the reader to [17]. Generally speaking, rigid registration of mono-modal images from the same patient a solved problem. Many methods, including the Fourier transform-based method or the voxelsimilarity-based method, can align images of this type with subpixel accuracy. For registering images with significant contrast differences, such as those from different modalities or acquired with different matching MR sequences, the most effective matching criterion is the mutual information metric. With the advance of the available computational power, registration of non-rigid monomodal or multi-modal images has become a hot research area in the past several years. One of the reasons what non-rigid registration gained popularity is that registration can be used to solve various image segmentation problems. This strategy is called atlas-based segmentation.

The challenges remaining for image registration include [12]:

- Interpolation effects: Mutual information is the most popular similarity measure for registering multi-modal images. However, together with most of the existing matching metrics, Mutual Information metric is sensitive to image interpolation artifacts, which limits the registration accuracy. To overcome this problem, special interpolators are needed.

- Non-rigid body registration: Nonrigid body image registration, in general, is still an open problem. Much work remains to develop practically useful deformable registration techniques.

- Registration assisted segmentation and segmentation guided registration: The traditional Atlas-based segmentation approaches takes a two stage philosophy: non-rigid registration between two images is firstly carried 
out, and then secondly the resulting transformation is applied to the atlas to get the segmentation for the input image. The prior information embedded in the atlas is totally neglected during the registration step. Registration assisted segmentation and segmentation guided registration are the directions worth pursuing.

\subsection{Chapter Outline}

The rest of the chapter is organized as follows: Section 2 reviews and analyzes the generation mechanism of the interpolation artifacts when using Mutual Information [15]. Section 3 reviews the related work for joint segmentation and registration problem. A variational framework that integrates prior segmentation information into the non-rigid registration procedure will be discussed. Section 4 concludes this chapter.

\section{Mutual Information Metric and Artifact Ef- fects [15]}

This section is dedicated to the first challenge we pointed out in the section 1.2: Interpolation effects. We will take Mutual-Information as an example to analyzes the generation mechanism of the artifacts inherent in the interpolation procedure. Remedies to reduce the artifacts will also be presented.

Consider two images $I_{r}(x, y)$ and $I_{f}(x, y)$. We designate $I_{r}$ as the reference image and $I_{f}$ as the floating image. Registration is to find the coordinate transformation, denoted as $T$, such that transformed floating image $I_{f}(T(x, y))$ is aligned with the reference $I_{r}(x, y)$. The alignment is usually obtained by optimizing a certain similarity metric. So normally a registration algorithm consists of three components [1]: a coordinate transform, a similarity criteria, and a numerical scheme to seek the optimum.

Mutual information is currently the most popular matching metric being used in handling the registration problem for multimodal images. The MI between two discrete random variables, $A$ and $B$, is defined as [6]:

$$
M I(A, B)=\sum_{a, b} p_{A B}(a, b) \log \frac{p_{A B}(a, b)}{p_{A}(a) \cdot p_{B}(b)}
$$

where $p_{A}(a), p_{B}(b)$ and $p_{A B}(a, b)$ are the marginal probability distributions 
and joint probability distribution, respectively. The relationship between MI and entropy is:

$$
M I(A, B)=H(A)+H(B)-H(A, B)
$$

with $H(A)$ and $H(B)$ being the entropy of $A$ and $B$, and $H(A, B)$ their joint entropy.

$$
\begin{aligned}
& H(A)=-\sum_{a} p_{A}(a) \log p_{A}(a) \\
& H(A, B)=-\sum_{a, b} p_{A B}(a, b) \log p_{A B}(a, b)
\end{aligned}
$$

Given a set of samples, there are several approaches to estimate the probability functions $p_{A B}(a, b)$, most notably the histogram-based method [5] and Parzen window method $[26,28]$. In this paper, we focus on histogram-based method because it's widely used in image registration. To register the images the mutual information is to be maximized.

The advances of mutual information based methods reside not only in the impressive accuracy in the reported registration experiments, but also the generality the MI methods can provide. Very few assumptions ever made regarding the relationship that exists between the image intensities, so mutual information is especially suited for multi-modality matching and that it is completely automatic.

Studholme et al. [20] pointed out the standard mutual information is sensitive to the field of view of the scanner used in the image acquisition, namely, with different choices of field of view, the maximization process may lead to an incorrect alignment. The authors then extended the mutual information to a normalized mutual information to alleviate this problem:

$$
N M I(A, B)=(H(A)+H(B)) / H(A, B)
$$

\subsection{Interpolation Artifact Effects}

For digital images $I_{r}(x, y)$ and $I_{f}(x, y)$ to be aligned, interpolation is necessary to evaluate the values of $M I\left(I_{r}(x, y), I_{f}(T(x, y))\right.$. A number of interpolators are available, including nearest neighbor $(\mathrm{NN})$, linear, cubic B-spline, Hamanning-windowed sinc and partial volume (PV) interpolators. Among them, PV is regarded as the best choice for MI-based metrics, as pointed out by several studies $[11,16]$.

Partial volume interpolation is not an interpolation in the ordinary sense. It is a strategy being used to update the joint histogram. As shown in Figure 3, 


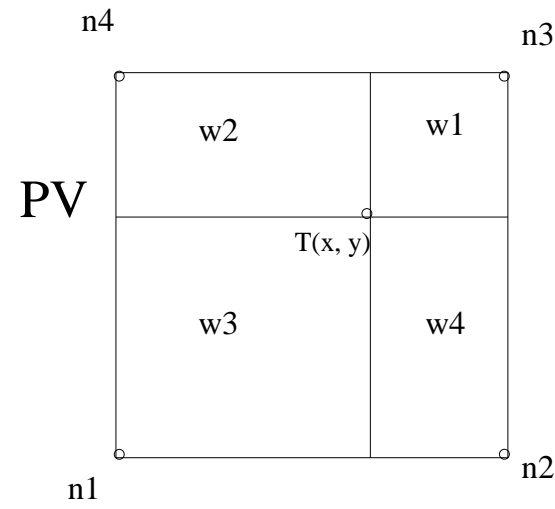

Figure 3: PV interpolation.

instead of interpolating the new intensity value under current transformation $T$, PV directly updates the histogram of the nearest neighbor bins with the same weights used in bilinear (for 2D) or trilinear (for 3D) interpolation.

In [19], Maintz et al. qualitatively explained the reason why artifacts are generated in partial volume interpolation process and verified their arguments through several well-designed experiments. While their work is very informative, we believe that a theoretically quantitative analysis concerning the generation mechanism of artifacts will be more instructive to guide the related research.

As interpolation affects the registration function of normalized MI and traditional MI in a similar way [19], we will construct our arguments based on traditional MI in this paper, but it should be noted that the conclusions also hold for normalized MI.

As given above, the mutual information $M I(A, T(B))$ consists of 3 terms: $H(A), H(B)$ and $H(A, T(B)) . H(A)$ is a constant. The computation of $H(T(B))$ is also affected by the interpolation effect, but in a much smaller extent. Figure 4 shows a pair of aligned MR/CT images and the associated marginal entropies, joint entropy and MI values as functions of translations up to \pm 7 pixel distances. As evident, the variation of $\mathrm{MI}$ is dominated by the changes in $H(A, T(B)) ; H(A)$ and $H(T(B))$ are close to constants. So from now on, we will focus only on $H(A, T(B))$.

Let's first consider the situation where the reference and floating images have exactly the same pixel sizes and the motion is limited to translations 

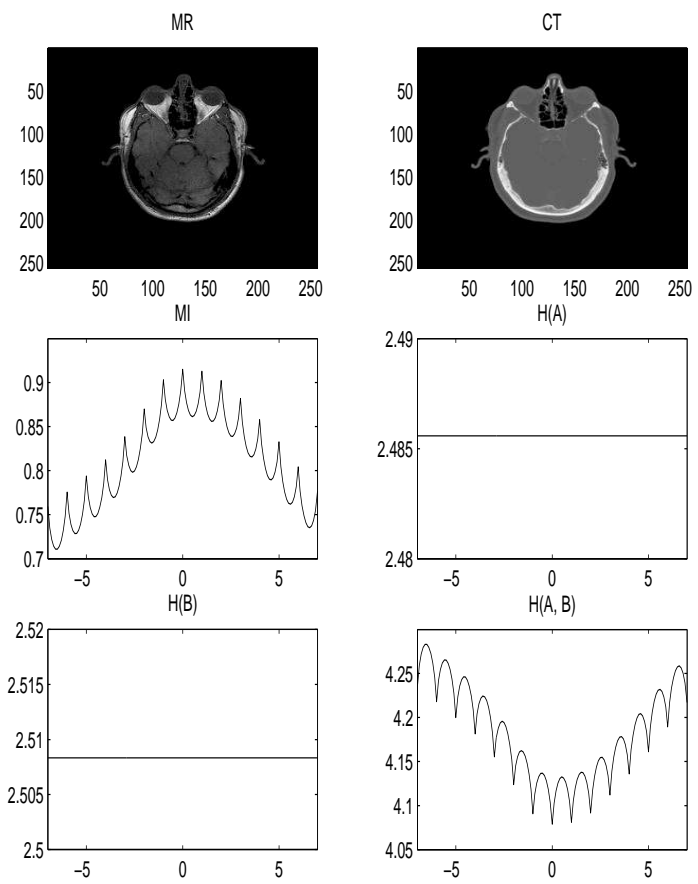

Figure 4: The mutual information value of a pair of multimodal images. Row 1 contains a pair of $\mathrm{MR} / \mathrm{CT}$ images (Available online at http://www.isi.uu.nl/Research/Registration/). Row 2 and row 3 shows the mutual information(MI),marginal entropies $(H(A)$ and $H(T(B)))$ and joint entropy $((H A, T(B)))$ values as functions of translations $t$ (up to \pm 7 pixels).

only. We use the CT image in Figure 4 as the reference, while MR is the floating image. Now let's analyze the variation of MI function between the two images when the floating image moves from the alignment position to 1 pixel away along $x$ axis.

Suppose at the alignment position (translation is equal to zero), a certain histogram bin his $(a, b)$ has a value of $M_{1}$. his $(a, b)$ records the total number of pixels in the image pair where the reference image has intensity $a$, and floating image $b$. Suppose when the translation is 1 pixel, his $(a, b)$ becomes $M_{2}$. Let's re-define the $h i s(a, b)$ to $h i s(a, b, t)$ to include the translation variable, then $h i s(a, b, 0)=M_{1}$ and his $(a, b, 1)=M_{2}$. In between, with the translation being $t$, there are a group of intensity grids $X 1=\left\{\left(x_{1}, y_{1}\right),\left(x_{2}, y_{2}\right) \ldots\right\}$ that were originally contributing to the bin $h i s(a, b)$, gradually wipe out their support 
when the floating image is moving. Let's call this group of grids as the movingout set, and let $A_{1}$ be the total number of these grids. Because the motion here is limited to translation only, all the grids in the moving-out set are withdrawing their contributions to the bin his $(a, b)$ at the same rate, as the translation increases from 0 to 1 . When the translation is 0 , each of them contribute an ' 1 ' to his $(a, b)$; when the offset is 1 , they do not have contribution any more. In between, the contribution of the each moving-out grid is $1-t$.

Similarly, there might be another group of grids $X_{2}$ (let A2 be the total number) that were not originally contributing to his $(a, b)$, start moving in to contribute to $h i s(a, b)$ as the translation increases from 0 to 1 . Their individual contribution to his $(a, b)$ is ' $t$ ' at translation $t$.

Overall, the combined effects of the moving-in and moving-out sets lead to the change of $h i s(a, b)$. So we have:

$$
A_{2}-A_{1}=M_{2}-M 1
$$

At translation t:

$$
\begin{aligned}
\operatorname{his}(a, b, t) & =M_{1}+A_{2} t-A_{1} t \\
& =M_{1}+(M 2-M 1) t \\
& =t M_{2}+(1-t) M_{1}
\end{aligned}
$$

So basically within interval $[0,1]$, the bin value $h i s(a, b, t)$ is a linear function of the offset variable t, denoted here by $f(t)$ :

$$
\begin{aligned}
& f(0)=M 1, \quad f(1)=M 2 \\
& f(t)=t f(1)+(1-t) f(0) .
\end{aligned}
$$

Since we use histogram to approximate distribution, the joint entropy of two images can be rewritten as $H(A, T(B))=-\sum_{a, b} h i s(a, b, t) \log h i s(a, b, t)$. As we know, the function $x \log x$, denoted by $g(x)$ here, is a convex function within the interval $(0,1]$ (note its second derivative is positive), i.e.:

$$
g\left(t x_{1}+(1-t) x_{2}\right) \leq t g\left(x_{1}\right)+(1-t) g\left(x_{2}\right)
$$

Therefore, the individual contribution of the bin his $(a, b, t)$ to the joint entropy $H(A, T(B))$ follows:

$$
\begin{aligned}
g(f(t)) & =g((1-t) f(0)+t f(1)) \\
& \leq(1-t) g(f(0))+t g(f(1))
\end{aligned}
$$


The inequality above indicates that each component of $H(A, T(B))$ is a convex function within $[0,1]$. Since the summation of convex functions is still a convex function, $\mathbf{H}(\mathbf{A}, \mathbf{T}(\mathbf{B}))=-\sum \sum \mathbf{g}(\mathbf{h i s}(\mathbf{a}, \mathbf{b}, \mathbf{t}))$, as a negative combination of certain number of convex functions, is a concave function in $[0,1]$. Correspondingly, the MI responses is a convex function in the same interval. This property can be easily extended the any intervals $[n, n+1]$ where $n$ is an integer. That's the reason the responses of $H(A, T(B))$ as a function of translation $t$ (Figure 4 ) bears a concave-shaped artifact within each integer interval.

If we take a closer look at the above analysis, we can find that the heart of the artifact generation mechanism lies in the following facts:

1. When translation is the only motion type, bilinear interpolation causes the bin values to change in a linear fashion with respect to displacement. In other words, due to the linear kernel used in PV interpolation, all the moving-in and moving-out grids contribute to the changes of the bin values at a synchronized pace.

2. $x \log x$ is a convex function.

As a consequence, a general guideline to reduce the artifact effects can be "to break the synchronization".

In addition, the following prediction can be made based on the above analysis:

- The artifact effect for pure rotations would be less severe than that of pure translations. This is because the moving-in and moving-out grids, under the pure rotation motion scenario, do not contribute to the change of histogram in a uniformly rate. Figure 5.a. shows the $H(A, T(B))$ values as a function for rotations (up to $\pm 15^{\circ}$ ). As evident, the responses for rotations are much smoother than the translation counterpart.

In the past years, several remedies for reducing the artifact effects have been proposed $[22,4]$, which either relies on resampling one of the input images into different grid sizes, or on applying higher-order functions as the interpolation kernel. Although a number of impressive results have been reported, we believe the analysis given in previous sections can provide a deeper insight about which kernel should be chosen and how it works. 

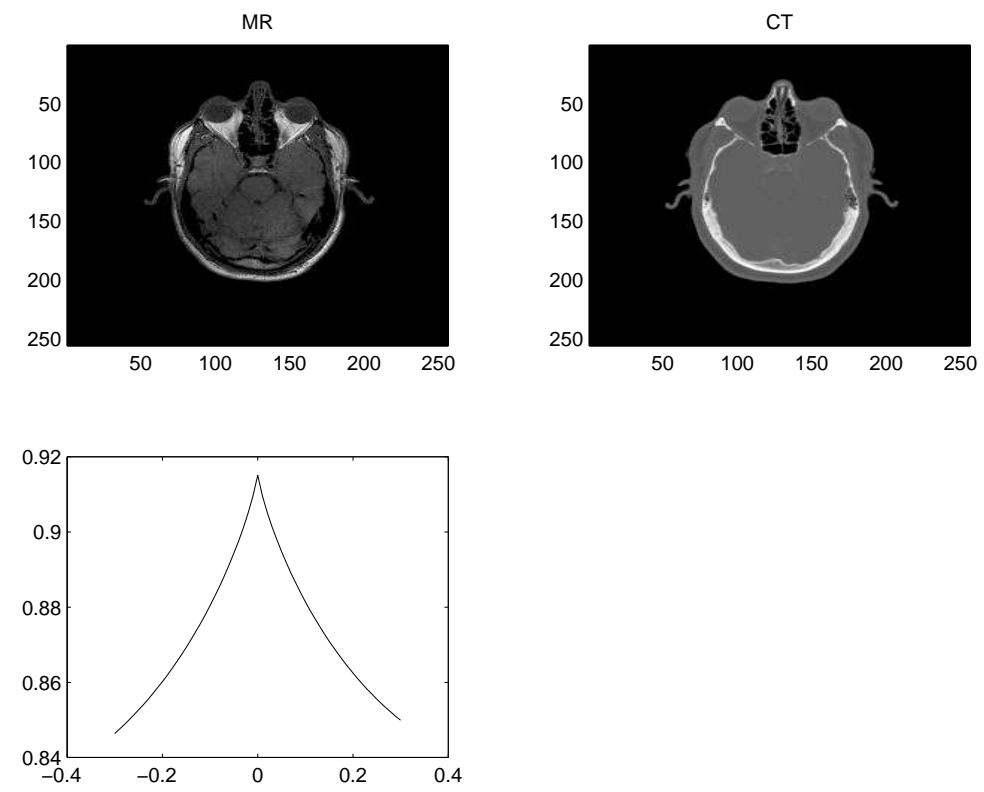

Figure 5: Row 1 contains a pair of MR/CT images (from the homepage of the Image Science Institute, Department of Medical Imaging. Available online at http://www.isi.uu.nl/Research/Registration/registration-frame.html). Row 2 shows the mutual information (MI) value as a function to rotaions $\left( \pm 20^{\circ}\right)$.

When bilinear function is used as the PV interpolation kernel, all the relevant grids contribute to the change of a histogram bin value at a synchronized pace. As we mentioned earlier, "to break the synchronization" is the key for reducing/removing the interpolation artifacts. For a new interpolation kernel to be chosen to avoid this translation-caused synchronization, two desired properties have to be satisfied:

1. The filter support width should be larger than 2. As being exemplified in bilinear interpolation, if the support comes only from the 4 surrounding grid points (filter width is 2 ), at the time that translation is the only motion form, regardless what interpolation kernel is being used, the changes of bin values will always take place in a synchronized fashion.

2. Uniform (linear) function should not be used as the kernel. If bilinear function is kept as the interpolation kernel, even with a broader support, e.g., 16 points (filter width $=4$ ) are used in updating the histogram bin 
values, due to the consistence existing in image intensities, synchronization is still likely to occur.

In a nutshell, broad-support non-uniform interpolation kernels should be chosen for the purpose of artifacts reduction. We now verify this thought by utilizing two non-uniform together with a uniform functions and observe their different performances. In the following experiments, we consider three interpolation kernels: cubic B-spline approximation [9], Gaussian function and super-linear interpolation.

The cubic B-spline approximation used in this paper has a filter support width of 4 , and the function kernel is given as follows:

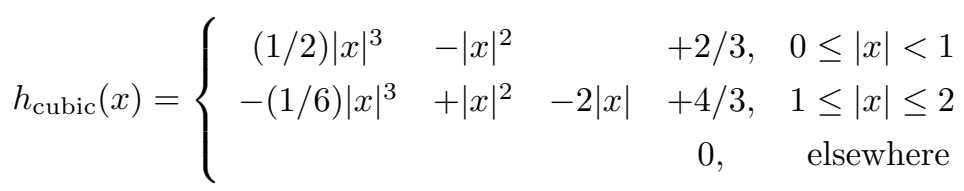

The Gaussian function used here also has a support of 4, and its standard deviation $\sigma$ is set to 1 . The kernel function is given:

$$
h_{\text {Gaussian }}(x)=\frac{1}{\sqrt{2 \pi} \sigma} \exp \left(-\frac{x^{2}}{2 \sigma^{2}}\right)
$$

The super-linear function is an interpolator built only for the purpose of verifying the second claim: "uniform function should not be used as the kernel". This filter has a support width of 4 , which is broader than the bilinear kernel, however it's still a uniform function.

$$
h_{\text {super-linear }}(x)=\left\{\begin{array}{cc}
1 / 2-|x| / 4, & 0 \leq|x| \leq 2 \\
0, & \text { elsewhere }
\end{array}\right.
$$

Figure 6 shows the above three interpolation kernels. Their corresponding MI responses with respect to the displacements along x-axis are given in Figure 7. The left-top subfigure of Fig. 7 is the artifact-filled MI response from the linear PV interpolation. MI response using cubic B-spline is given in the righttop subfigure. Due to the non-uniformity of the cubic B-spline function, for each transformed pixel, its surrounding 4 points contribute to the histogram bin values in a different pace with the outside 12 points do. As a consequence, synchronization is broken and the artifact effect is reduced. As evident, the MI response curve for the cubic B-spline kernel is quite smooth. Same arguments can be applied to the Gaussian kernel function. As shown in the left-bottom 

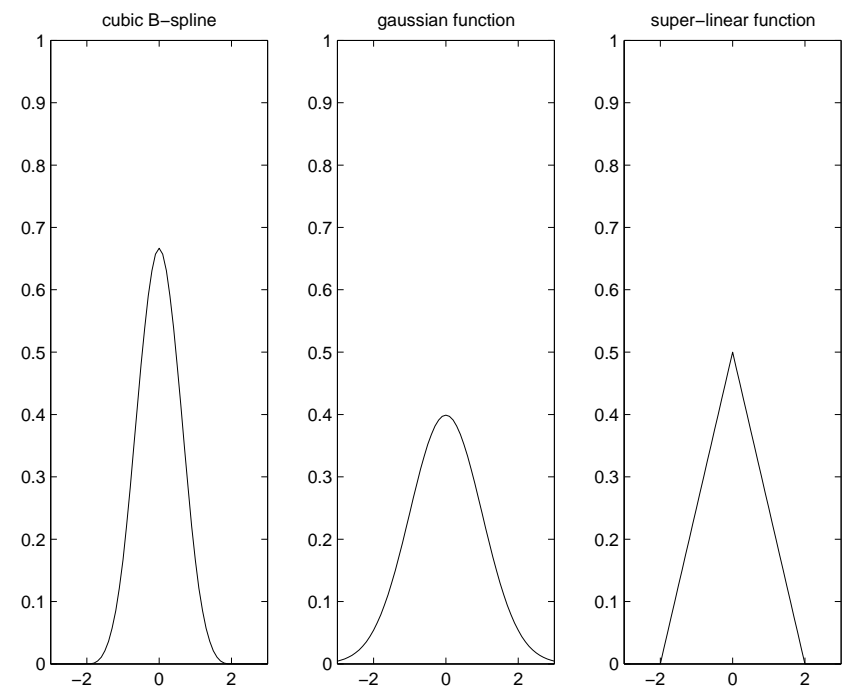

Figure 6: Three interpolation kernels. Left: Cubic B-spline function; middle: Gaussian function; right: super-linear function.

subfigure, artifact patterns almost disappear. One should note that the MI optima for both cubic B-spline and Gaussian, as evident in the figure, have not been visibly dislocated, which implies the ultimate registration estimation can still be very accurate even though both Cubic B-spline and Gaussian are "blurring" filters. Right-bottom subfigure shows the registration response from super-linear interpolator. Comparing with the narrower-support bilinear function, super-linear brings more point to smooth up the interpolation procedure, thus the artifact pattern becomes less severe. However, as super-linear is still a uniform kernel, the resulting response pattern is far from being artifact-free.

\subsection{Experiment Results}

In this section, we demonstrate the robustness property of the new interpolation kernels proposed in the previous section. All the examples contain synthesized miss-alignments applied to a pair of aligned CT/MR images. This pair of $\mathrm{CT} / \mathrm{MR}$ images were obtained from the homepage of the Image Science Institute at the University of Medical Center Utrecht (Available online at http://www.isi.uu.nl/Research/ Registration/registration-frame.html).

The experiments are designed as follows: with a 2D CT slice as the refer- 

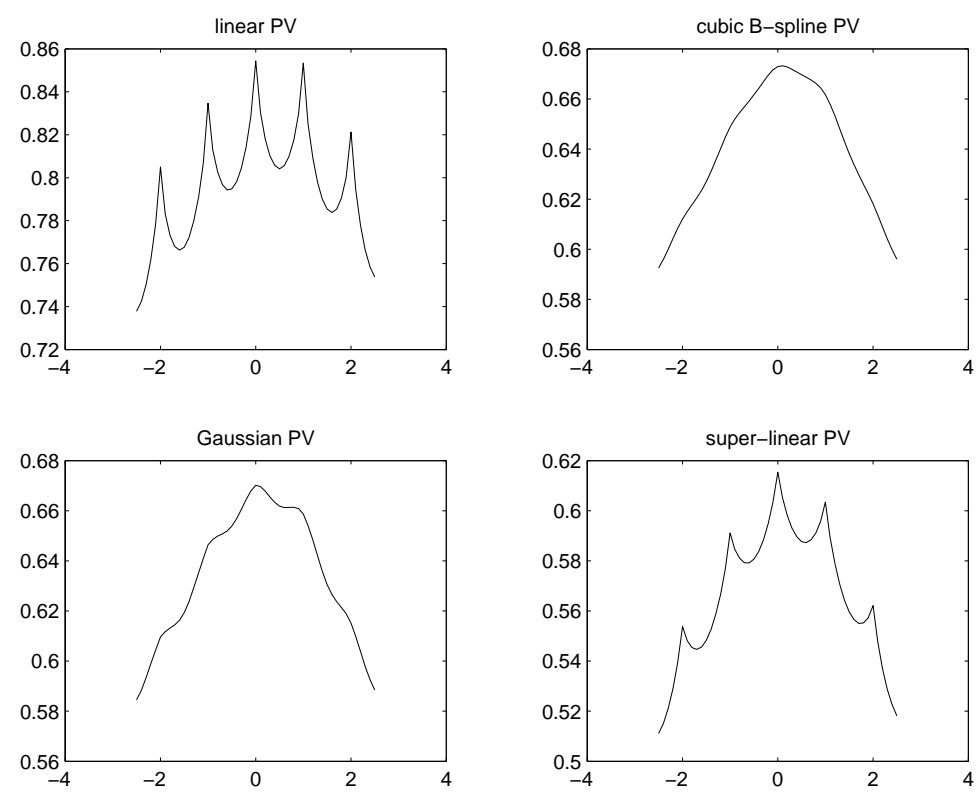

Figure 7: Mutual information responses with respect to the translations along $\mathrm{x}$-axis. Left-top: values for Bi-linear interpolator; Right-top: for Cubic B-spline function; Left-bottom: for Gaussian function; Right-bottom: for super-linear function. 
ence image, the floating image is obtained by applying a rigid transformation to a previously aligned 2D MR image. With 15 randomly generated rigid transformations, we applied 3 different functions: bi-linear, Cubic B-spline and Gaussian, as the interpolation kernels to estimate the motion parameters. These transformations are normally distributed around the values of $\left(10^{\circ}, 10\right.$ pixel, 10 pixel $)$, with standard deviations of $\left(3^{\circ}, 3\right.$ pixel 3 pixel) for rotation and translation in $x$ and $y$ respectively.

Table 1 depicts the mean and standard deviation of the estimation errors obtained from the 3 different interpolation kernels. In each cell, the leftmost value is the rotation angle (in degrees), while the right two values show the translations in $\mathrm{x}$ and $\mathrm{y}$ directions respectively. Out of the 15 trials, the linear PV method failed 5 times while the Cubic B-spline and Gaussian interpolations all succeeded ("failed" here means that the results had unacceptably large errors). If we only count the cases which gave reasonable results, as shown in the first (for Cubic B-spline ), second (for Gaussian function) and third (for linear PV) rows, our approach and the traditional MI have comparable performances, all being very accurate. Note that Powells method was used as the optimization scheme in these experiments.

\begin{tabular}{|c|rcc|ccc|}
\hline \hline & \multicolumn{3}{|c|}{ mean } & \multicolumn{3}{c|}{ standard deviation } \\
\hline Cubic B-spline & $0.149^{\circ}$ & -0.293 & 0.148 & $0.049^{\circ}$ & 0.091 & 0.198 \\
\hline Gaussian & $0.056^{\circ}$ & 0.250 & 0.128 & $0.032^{\circ}$ & 0.201 & 0.218 \\
\hline Bi-linear & $0.087^{\circ}$ & 0.293 & 0.383 & $0.031^{\circ}$ & 0.121 & 0.129 \\
\hline
\end{tabular}

Table 1: Comparison of estimation errors for rigid motion between Cubic Bspline, Gaussian and Bi-linear functions as the interpolation kernels.

Figure 8 depicts an example of the registration results. Fig 8.a is the reference CT image. Fig 8.b and Fig 8.c are the floating MR images, before registration and after registration, respectively. An edge map of the reference CT image is superimposed on the transformed floating image. As evident, the registration is visually quite accurate. 

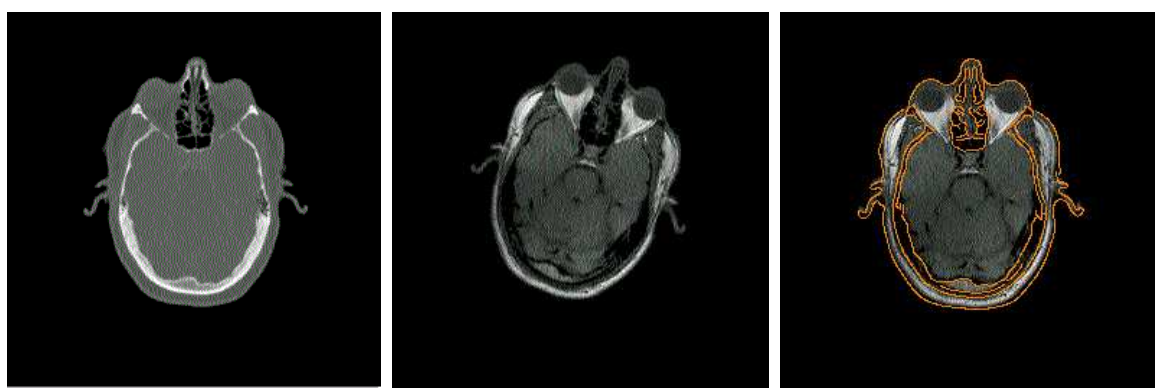

Figure 8: An example of the registration results. The leftmost is the reference CT image; middle is the floating MR image prior to registration and the rightmost is the MRI after registration. Images were obtained from the homepage of the Image Science Institute at the University of Medical Center Utrecht (Available online at http://www.isi.uu.nl/Research/ Registration/registrationframe.html).

\section{Segmentation-Guided Deformable Image Reg- istration Frameworks [14]}

In this section, we address the second and third challenges we pointed out in the section 1.2: how to integrate segmentation and registration into a unified procedure so that the prior information embedded in both processes can be better utilized.

Registration and segmentation are two most fundamental problems in the field of medical image analysis. Traditionally, they were treated as separate problems, each with numerous solutions proposed in literature. In recent years, the notion of integrating segmentation and registration into a unified procedure has gained great popularity, partially due to that more and more practical problems, e.g., atlas-based segmentation, subsume both segmentation and registration components.

Yezzi et al. [30] pointed out the interdependence existing in many segmentation and registration solutions, and a novel geometric, variational framework was then proposed that minimizes an overall energy functional involving both pre and post image regions and registration parameters. Geometrical parameters and contour positions were simultaneously updated in each iteration, and segmentations were obtained from the final contour and its transformed 
counterpart. While this model and its variants [23, 24] are enlightening and pave a promising way towards unifying registration and segmentation, their applicability range is either limited to relatively simple deformation type [30] (rigid/affine), or to relatively simple input images [23, 24].

Vemuri et al. [32] propose a segmentation + registration model to solve the atlas-based image segmentation problem where target image is segmented through the registration of the atlas to the target. A novel variational formulation was presented which put segmentation and registration processes under a unified variational framework. Optimization is achieved by solving a coupled set of nonlinear PDEs.

Another segmentation + registration model proposed by Noble et al. [18] seeks for the best possible segmentation and registration from the maximum a posteriori point of view. Improvements in accuracy and robustness for both registration and segmentation have been shown, and potential applications were identified. This model is primarily designed for combining segmentation and rigid registration. While non-rigid algorithm was also implemented, the motion field estimation is based on block-matching of size $(7 \times 7)$, which is not dense enough for most non-rigid registration applications.

\subsection{Proposed Registration Method}

Inspired by the above-mentioned approaches, the work presented in this section is aimed to establish a segmentation assisted framework to boost the robustness of non-rigid image registration. Segmentation information is integrated into the process of registration in leading to a more stable and noise-tolerant shape evolution, while a diffusion model is used to infer the volumetric deformation across the image.

Our approach differs from other models in that we use a unified segmentation + registration energy minimization formulation, and the optimization is carried out under the level set framework. A salient feature of our model is its robustness against input image noise. We present several 2D examples on synthetic and real data in the implementation results section.

\subsection{Segmentation Guided Registration Model}

Commonly, the basic input data to a registration process are two images: one is defined as fixed (or target) image $I_{1}(X)$ and the other as the moving (or 
source) image $I_{2}(X)$. In addition to these two image, our model requires a segmentation of the fixed image, indicting a studying area of $I_{1}(X)$, as another input component.

Let $C$ be the boundary curve of the segmentation. We denote by $C_{i n}$ and $C_{\text {out }}$ representing the inside and outside areas of the curve $C$. Let $C_{1}$ and $C_{2}$ be the average values for $C_{i n}$ and $C_{\text {out }}$ respectively.

The contour $C$ can be either input by user or derived from a training set. We assume that the region captured by $C$ contains a single object of the fixed image, therefore the intensity values of both inside and outside of the region should be relatively homogenous. Suppose the fixed and moving images are well corresponded, then, at the time a perfect alignment is achieved, the intensities in the warped moving image should also be relatively uniform within both $C_{\text {in }}$ and $C_{\text {out }}$. This observation provides the justification for our model, which is designed based on following considerations:

In addition to the set of forces generated by intensity similarity measure (e.g., SSD) to warp the moving image toward the target, another set of forces, derived from the region homogeneity constraint, should be utilized to pull the moving image toward the correct alignment. This set of forces can provide an extra guideline for the registration process to avoid local energy optima, which is especially helpful when input images are noisy.

Our solution to the registration problem is to minimize the following energy,

$$
\begin{aligned}
E(V)= & \int_{\Omega}\left[I_{1}(X)-I_{2}(X+V(X))\right]^{2} d X \\
& +\lambda_{1} \int_{C_{\text {in }}}\left[I_{2}(X+V(X))-C_{1}\right]^{2} d X \\
& +\lambda_{2} \int_{C_{\text {out }}}\left[I_{2}(X+V(X))-C_{2}\right]^{2} d X \\
& +\lambda_{3} \int_{\Omega}\|\nabla V(x)\|^{2} d X
\end{aligned}
$$

where $\Omega$ is the image domain and $V(X)$ denotes the deformation field. $\lambda_{1}, \lambda_{2}$ and $\lambda_{3}$ are three constant parameters that weight the importance of each term in the optimization energy. The $\int\left[I_{1}(X)-I_{2}(X+V(X))\right]^{2} d X$ term provides the main force for matching two images, while $\int\left[I_{2}(X+V(X))-C_{1}\right]^{2} d X$ and $\int\left[I_{2}(X+V(X))-C_{2}\right]^{2} d X$ terms allow the prior segmentation to exert its 
influence, aiming to enforce the homogeneity constraints. $\int\|\nabla V(X)\|^{2} d X$ is a diffusion term to smooth the deformation field.

\subsubsection{The level set formulation of the model}

Functional (17) can be minimized under the level set framework. Introduce a continuous function $\phi: \Omega \rightarrow R$, so $C=\{(X) \in \Omega: \phi(X)=0\}$, and we choose $\phi$ to be positive in $C_{\text {in }}$ and negative in $C_{\text {out }}$. For the level set formulation, we adopt the model presented in Chan et al. [3]. The new energy, still denoted by $E(V)$ is changed to:

$$
\begin{aligned}
E(V)= & \int_{\Omega}\left[I_{1}(X)-I_{2}(X+V(X))\right]^{2} d X \\
& +\lambda_{1} \int_{\phi \geq 0}\left[I_{2}(X+V(X))-C_{1}\right]^{2} d X \\
& +\lambda_{2} \int_{\phi<0}\left[I_{2}(X+V(X))-C_{2}\right]^{2} d X \\
& +\lambda_{3} \int_{\Omega}\|\nabla V(X)\|^{2} d X
\end{aligned}
$$

Using the Heaviside function $H$ defined by:

$$
H(x)= \begin{cases}1 & \text { if } x \geq 0 \\ 0 & \text { if } x<0\end{cases}
$$

and the one-dimensional Dirac measure $\delta$ function defined by:

$$
\delta(x)=\frac{d}{d x} H(x)
$$

The second and third terms can be rewritten as:

$$
\begin{aligned}
E(V)= & \int_{\Omega}\left[I_{1}(X)-I_{2}(X+V(X))\right]^{2} d X \\
& +\lambda_{1} \int_{\Omega}\left[I_{2}(X+V(X))-C_{1}\right]^{2} H(\phi(X)) d X \\
& +\lambda_{2} \int_{\Omega}\left[I_{2}(X+V(X))-C_{2}\right]^{2}(1-H(\phi(X))) d X \\
& +\lambda_{3} \int_{\Omega}\|\nabla V(X)\|^{2} d X
\end{aligned}
$$

The Euler-Lagrange equation of the functional (19) is given by: 


$$
\begin{aligned}
\frac{\partial E}{\partial V}= & 2\left(I_{1}(X)-I_{2}(X+V)\right)\left(-\nabla I_{2}(X+V)\right) \\
& +2 \lambda_{1}\left(I_{2}(X+V)-C_{1}\right) \nabla I_{2}(X+V) \cdot H(\phi(X)) \\
& +\lambda_{1}\left(I_{2}(X+V)-C_{1}\right)^{2} H^{\prime}(\phi(X)) \cdot \nabla \phi(X) \\
& +2 \lambda_{2}\left(I_{2}(X+V)-C_{2}\right) \nabla I_{2}(X+V) \cdot(1-H(\phi(X))) \\
& +\lambda_{2}\left(I_{2}(X+V)-C_{2}\right)^{2}\left(-H^{\prime}(\phi(X)) \cdot \nabla \phi(X)\right) \\
& +\lambda_{3} \nabla^{2} V
\end{aligned}
$$

where

$$
\begin{gathered}
C_{1}=\frac{\int_{\Omega} I_{2}(X+V) H(\phi(X+V) d X}{\int_{\Omega} H(\phi(X+V)) d x d y} \\
C_{2}=\frac{\int_{\Omega} I_{2}(X+V)(1-H(\phi(X+V)) d X}{\int_{\Omega}(1-H(\phi(X+V))) d x d y}
\end{gathered}
$$

The level set function being used in this paper is $\phi(X, 0)=D(X)$, where $D(X)$ is the signed distance from each grid point to the zero level set $C$. This procedure is standard, and we refer the reader to [21] for details.

\subsection{Experimental Results}

In this section, we demonstrate the improvement made by our algorithm, with synthetic as well as real data sets. In both cases, we compare the results using our model with that of using the popular Demons algorithm. We apply both schemes to the same sets of data.

The synthetic data example contains a pair of synthetically generated images, where the fixed image was generated from the moving by a known nonrigid field. Zero-mean Gaussian noise was then added to each image. The standard deviation is 20. Fig 9.a and 9.b show the two images. In the following examples, we chose the constants $\lambda_{1}=0.1, \lambda_{2}=0.1$ and $\lambda_{3}=1$, respectively.

The segmentation of the fixed image was manually obtained, as superposed on the moving image in Fig 9.c. Two registration approaches: the Demons algorithm as well as our segmentation-guided registration model are then applied. The Demons algorithm being used here relies purely on intensity for registration. 


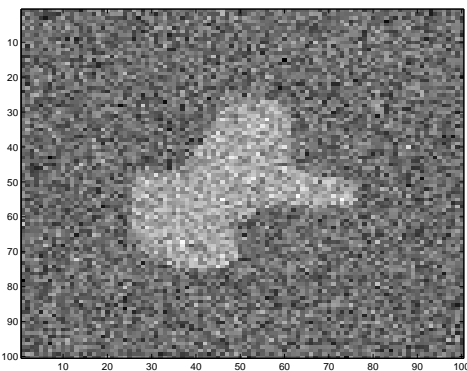

(a)

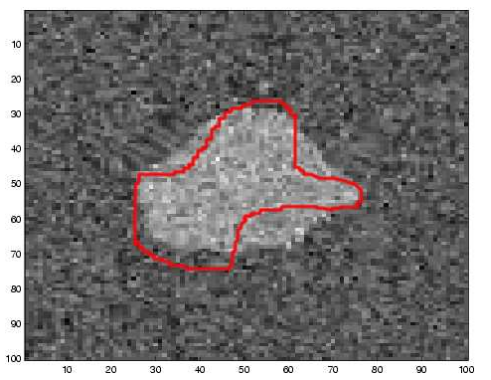

(c)

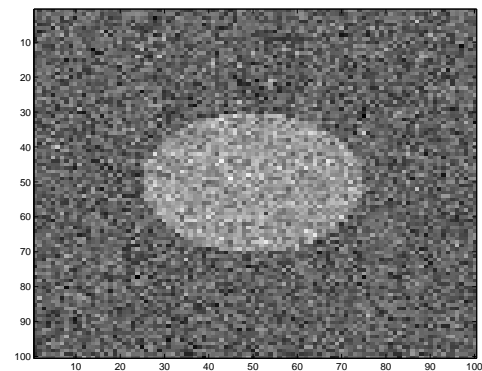

(b)

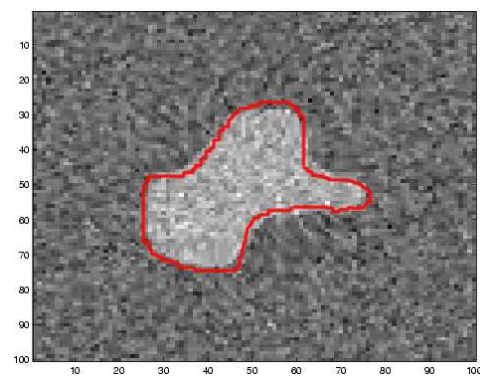

(d)

Figure 9: Registration results for image set 1. First row:(a) the fixed image and (b) the moving image. Second row: the registration result of (c) using the Demons algorithm, and (d) using our segmentation guided registration model. The edge map from the fixed image is superposed.

The results are shown in the second row of Figure 9. Fig 9.c is the transformed moving image from the Demons algorithm, after the registration is finished. Fig 9.d shows the result of our model. As evident, the Demons algorithm had trouble in warping the moving image to a perfect matching, which is partially due to the numerous local energy minima resulted from the huge amount of noise existing in the images. However, the registration result generated from our model is quite accurate, which indicates that the integrated segmentation information is very helpful in pulling the moving image towards a correct matching.

We designed and carried out a similar experiment on a pair of MRI brain slices. The images were obtained from the Davis-Mills Magnetic Resonance Imaging and Spectroscopy Center (MRISC) at University of Kentucky. The 


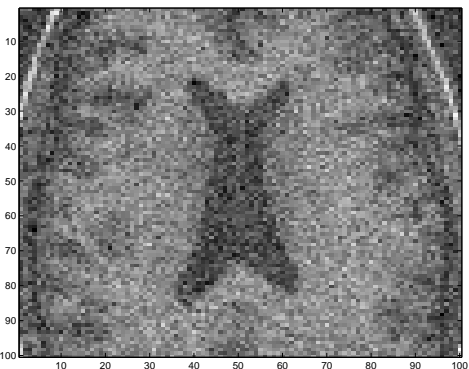

(a)

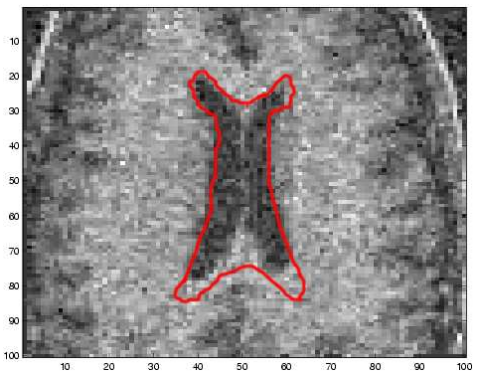

(c)

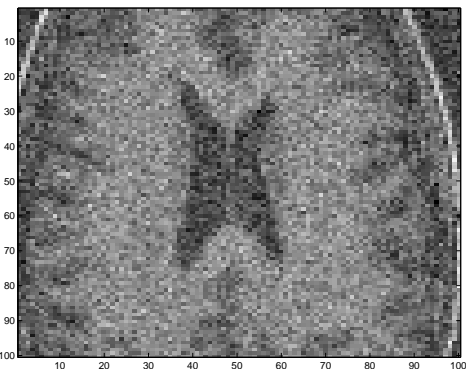

(b)

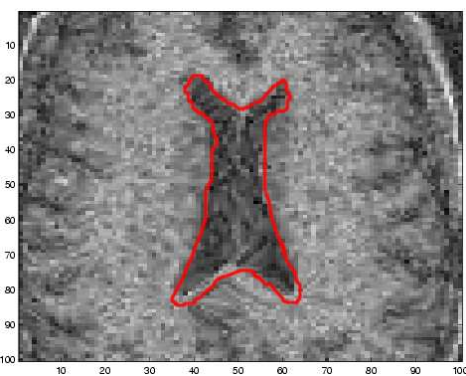

(d)

Figure 10: Registration results for image set 2 (images were obtained from the Davis-Mills Magnetic Resonance Imaging and Spectroscopy Center (MRISC) at University of Kentucky). First row:(a) the fixed image and (b) the moving image. Second row: the registration result of (c) using the Demons algorithm, and (d) using our segmentation guided registration model.

two slices have substantial disparity in shape of the ventricles, which is the region of interest. Figure 10 shows the images and results. Fig 10.a and 10.b are the fixed and moving images respectively. Fig 10.c and 10.d depict the results from the Demons algorithm (10.c) and our segmentation guided registration model (10.d). As evident, the former model fails to transform the ventricle area into a desired position, while the latter accurately achieves the registration goal. 


\section{Discussion and Conclusions}

As we mentioned in the introduction section, rigid registration for mono-modality images is fundamentally a solved problem, but not the case for multi-modality images. Methods based on statistical similarity measures, such as Mutual Information, Joint Intensity Distributions, Correlation Ratio etc., are currently the most popular solutions, but all of them suffer from the interpolation artefact problem.

In section 2, we quantitatively analyze the generation mechanism of the interpolation artifacts [15]. We conclude that the combination of linear interpolation kernel and translation-only motion type leads to the generation of the artifact pattern. As a remedy we propose to use non-uniform interpolation functions in estimating the joint histogram. Cubic B-spline and Gaussian interpolators are compared and we demonstrate the improvements via experiments on misalignments between $\mathrm{CT} / \mathrm{MR}$ brain scans.

Though successful in analyzing the $P V$ interpolation, we have to point out that linear interpolation is a more widely used interpolator than $P V$ in many image processing tasks, such as segmentation, transformation etc. MI artifacts also exist for Linear interpolation. Several studies [5, 19] have shown that the artifact patterns generated from linear interpolation is quite in the contrast to those of PV: linear interpolation tend to generate concave registration functions within the integer intervals. An intuitive explanation is that Linear interpolation blurs the intensity values, which likely reduces the dispersion of the image histogram.

All in all, the artifacts generated by these two major interpolation schemes reflect a very general phenomenon: in image registration, when the algorithm involves the combination of a specific interpolation method and a non-linear measure based on the joint histogram of the image, serious artefact in the registration function would often be resulted. Further research on the following topics are worth exploring:

- What would be the best (in term of accuracy) interpolation scheme for Mutual-Information based image registration?

- What are the artefact patterns for other popular similarity measures, such as Correlation Ratio (CR), Local Correlation (LC) etc?

- Is there a general and systematic solution to this entire interpolation 
artefact problem?

Comparing with rigid registration, to recover non-rigid deformation between two or multiple images are far more complicated problem, from both theoretical and computation point of views. Unifying/combing registration with segmentation provides a promising direction to eventually better solve this problem.

In section 3.2, we present a segmentation-guided non-rigid registration framework [14], which integrates the available prior shape information as an extra forces to lead to a noise-tolerant registration procedure. Our model differs from other methods in that we use a unified segmentation + registration energy minimization formulation, and the optimization is carried out under level-set framework. We showed the improvement made with our model by comparing the results with that of the Demons algorithm. To explore other similarity metrics under the same framework to handle more complicated inputs will be the focus of our future work.

Comparing with the registration algorithms that purely rely on intensity correspondence, our segmentation-guided registration approach has a particular advantage of being robust. However, limitations of our technique also exist, and one of them is that we assume the segmentation of the reference image is available before the registration is carry out. In many atlas-based applications, certain prior segmentation/shape is available, but it's not necessary the one exactly generated from the reference image. Such prior shapes are more likely obtained from certain previous population analysis, bearing more statistical commonality than precise details. A novel approach to interpret the commonality can be found in [27]. They model the statistical shape information of a training set as a sparse group of critical points, each of which can provide an individual force to lead a smoother and more consistent registration process. A number of experiments performed on both synthetic and real images show the beneficial effects of the statistical boundary information in improving registration robustness. However, it's foreseeable that these methods would produce less smooth results for noisy inputs if the boundary points are not sampled densely enough. All in all, how to better interpret and integrate available statistical information, and more ambitiously, how to unify segmentation and registration into a single procedure are both challenging research directions worth exploring. 


\section{References}

[1] J. L. Barron, D. J. Fleet, and S. S. Beauchemin, "Performance of Optical Flow Techniques," Intl. J. Comput. Vision, 1(12):43-77,1994.

[2] Simulated brain database [Online]. Available : http://www.bic.mni.mcgill.ca/brainweb/

[3] T. Chan, L. Vese, "An Active Contour Model without Edges" IEEE Trans. Image Processing , vol. 10, no. 2, pp. 266-277.

[4] H. Chen and P. K. Varshney, "Mutual Information-based CT-MR Brain Image Registration Using Generalized Partial Volume Joint Histogram Estimation", IEEE Trans. on Medical Imaging, Vol. 22, No. 9, Sept. 2003. pp. 1111 - 1119.

[5] A. Collignon, F. Maes, D. Delaere, D. Vandermeulen, and P. S. ang G. Marchal, "Automated multimodality image registration using information theory," Proc. IPMI,Y.J.C.Bizais, Ed., pp. 263-274,1995.

[6] Thomas M. Cover, Joy A. Thomas, Elements of Information Theory, John Wiley and Sons, 1991.

[7] J. P. Thirion, "Image matching as a diffusion process: an analogy with Maxwell's demons", Medical Image Analysis, Volume 2, Issue 3, September 1998, pp 243260 .

[8] B. Fischer and J. Modersitzki, "Image registration using variational methods", Visual Analysis, Image Based Computing and Applications workshop.

[9] T. M. Lehmann, C. Gonner and K. Spitzer, "Survey: Interpolation Methods in Medical Image Processing", IEEE Trans. on Medical Imaging, Vol. 18, No. 11, November 1999. pp. 1049-1075.

[10] M. Leventon and W. E. L. Grimson, "Multi-modal volume registration using joint intensity distributions," in MICCAI 1999.

[11] X. Ji, H. Pan and Z.P. Liang, "A region-based mutual information method for image registration", Proc. 7th Intl. Soc. Magn. Reson. Med., vol 3, pp 2193, May, 1999.

[12] E. Haacke, Zhi-Pei Liang, "Challenges of imaging structure and function with MRI", IEEE Engineering in Medicine and Biology, Sept/Oct., 2000. pp 55 - 62.

[13] B. Likar and F. Pernus, "A hierachical approach to elastic registration based on mutual information", Image and Vision Computing, 19, 2001, pp. 33-44. 
[14] Jundong Liu, Yang Wang, Junhong Liu, "A Unified Framework for Segmentation-Assisted Image Registration", 7th Asian Conference on Computer Vision, Hyderabad, India, January 13-16, 2006. Proceedings, Part II Lecture Notes in Computer Science, Volume 3852 / 2006, pp. 405-414.

[15] Jundong Liu, Mingxiu Wei, Junhong Liu, "Artifact reduction in mutualinformation-based CT-MR image registration", Proc. SPIE Vol. 5370, p. 11761186, Medical Imaging 2004: Image Processing; J. Michael Fitzpatrick, Milan Sonka; Eds.

[16] J. B. A. Maintz, P. A. van den Elsen and M. A. Viergever, "Comparison of Edge-based and Ridge-based Registration of CT and MR brain images," Medical Image Analysis, 1(2), pp. 151-161, 1996.

[17] J.B. Maintz and M. A. Viergever, "A Survey of Medical Image Registration," MedIA Vol. 2, pp. 1-36,1998.

[18] P.P. Wyatt and J.A. Noble, "MAP MRF joint segmentation and registration of medical images", Medical Image Analysis, vol. 7, pp. 539-552, 2003.

[19] J. Pluim, J. Maintz and M. Viergever, "Interpolatioin artefacts in mutual information-based image registration," Computer Vis Image Underst, vol. 77, pp 211-232, 2000

[20] C. Studholme, D. Hill and D. Hawkes, "An overlap invariant entropy measure of 3D medical image alignment," Pattern Recognition, Vol. 32, pp. 71-86,1999

[21] M. Sussman, P. Smereka and S. Osher, "A Level Set Approach for Computing Solutions to Incompressible Two-Phase Flow". Journal of Computational Physics, vol. 114, pp. 146-159, 1994.

[22] J. Taso, "Interpolation Artifacts in Multimodality Image Registration Based on Maximization of Mutual Information", IEEE Trans. on Medical Imaging, Vol. 22, No. 7, July, 2003. pp 845-864.

[23] G. Unal, G. Slabaugh, A. Yezzi and J. Tyan, ” Joint segmentation and non-rigid registration without shape priors", Siemens Technical Report SCR-04-TR-7495, 2004.

[24] G. Unal and G. Slaaugh, "Coupled PDEs for Non-Rigid Registration and Segmentation", CVPR 2005, San Diego, CA, 2005.

[25] B. C. Vemuri, S. Huang, et.a.l., "An efficient motion estimator with application to medical imaging," Med. Image Anal., 2(1), pp. 79-98, 1998. 
[26] P. A. Viola and W. M. Wells, "Alignment by maximization of mutual information," in Fifth ICCV, MIT, Cambridge, MA, pp. 16-23, 1995

[27] Y. Wang and L. H. Staib, "Physical model-based non-rigid registration incorporating statistical shape information," Medical Image Analysis (MedIA), vol. 4, no. 1 , pp. $7-20,2000$.

[28] W. M. Wells III, P. Viola, and H. Atsumi, "Multi-modal Volume Registration by Maximization of Mutual Information," Medical Image Analysis, 1(1), pp. 35-51, 1997.

[29] R. Woods, J. Maziotta and S. Cherry "MRI-PET registration with automated algorithm," J. Comput. Assis. Tomogr., 17, pp. 536-546, 1993.

[30] A. Yezzi, L. Zollei and T. Kapur, "A Variational Framework for Joint Segmentation and Registration", Medical Image Analysis, pp 171-185, June 2003.

[31] S. Zhu and A. Yuille, "Region Competition, Unifying Snakes, Region Growing, and Bayes/MDL for Multibank Image Segmentation", IEEE TPAMI, Vol. 18, No. 9, Sept, 1996.

[32] B. Vemuri, Yun Chen and Z. Wang, "Registration Assisted Image Smoothing and Segmentation". ECCV (4) pp. 546-559, 2002. 